\title{
Políticas de información y biblioteca, comenzando por el principio
}

\author{
Horacio Cardenas Zardoni \\ Universidad Autónoma de Coahuila - UADEC, México
}

OPINION

\begin{abstract}
Resumen
Las políticas de la información no ocurren en abstracto, como parte de una teoría o de las ciencias de la información, tampoco se dan aisladamente, desvinculadas de la acción organizada de la sociedad. Si bien el sector productivo puede tener una perspectiva clara de a dónde quiere llegar, el ámbito de las políticas de la información corresponde a los gobiernos nacionales. Las políticas de información para alcanzar los objetivos que se ha planteado la sociedad de la información, necesariamente tienen que inscribirse como parte de las políticas públicas, especialmente las relacionadas con las estrategias para el desarrollo social, y articularse con el esfuerzo educativo de cada país. Siendo la biblioteca la entidad que siempre ha fungido como repositorio, y que tiene la función de hacer accesible aquel material que satisfaga las necesidades de información de la población, debe ser contemplada como parte integral de las políticas públicas y de información.
\end{abstract}

Palabras clave

Políticas de información ; Políticas públicas ; Desarrollo social ; Políticas educativas ; Bibliotecas

Information policies and libraries, beginning at the beginning

\begin{abstract}
Information policies do not occur in the abstract, as part of a theory or science of information, they do not exist in isolation, isolated from the organized action of society as a whole. While the productive sector may have a clear perspective of where they want to go, the field of information policy corresponds to national governments. In order to achieve the goals that Information policy has raised in the information society, they necessarily have to enroll as part of public policies, especially those related to social development strategies, and with each country's educational effort. The library as a social entity has always served as a repository, making available materials that meet the information needs of the population, as such it should be seen as an integral part of public and information policy.
\end{abstract}

Keywords

Information policy ; Public policy ; Development ; Educational policy ; Libraries

\section{Introdución}

Son muchos los autores que en años recientes han externado opiniones en torno a que las políticas de información se han convertido en un elemento de discurso cada vez más utilizado en los más diversos ámbitos, sin que detrás de las frecuentes referencias exista la conexión que debería haber con un creciente corpus teórico, con los deseables acuerdos con los científicos de la información y las disciplinas sociales, y muy particularmente, con una manifiesta direccionalidad hacia las áreas del ejercicio de las políticas públicas a cargo de los gobiernos. 
Las políticas de información, que habrían sido concebidas como un recurso orientador de la acción de las organizaciones, de las empresas y de los gobiernos hacia un objetivo claro y deseablemente concertado, en realidad son directrices poco efectivas, pues detrás de la declaración de apego a las mismas, hay un sustento muy limitado sin considerar que al igual que ocurre con los individuos, también estas instancias son susceptibles de caer en distraer el esfuerzo de los recursos en los diferentes aspectos del siempre creciente cúmulo de información, no pertinentes a la gran meta inicial, que se planteó para alcanzar a través específicamente de las políticas de información

En el presente artículo se procura establecer la importancia que tiene el amplio y genérico concepto de políticas de información para un tema de práctica cotidiana de manejo de la misma, el de las bibliotecas, entidades que deberían ser consideradas como de elección como herramienta institucional para que aquellas estuvieran en condiciones de cumplir con cualesquiera que sean las altas expectativas de quienes las formulan.

\section{Un poco de historia}

Sandra Braman sitúa el surgimiento de las políticas de información como un campo disciplinar hacia las últimas décadas del siglo pasado, como una manifestación del proceso de transformación de la sociedad industrial, a una sociedad basada en la información, y a la cual se ha denominado precisamente de esa manera, lo cual por sí mismo plantea un nivel de exigencia nunca antes visto, pues si bien todos los seres humanos forman parte de una sociedad, y una alta proporción pertenecía a las sociedades industriales, la de la información por su misma naturaleza ha de incluir a la totalidad del género humano, ideal que aún está lejos y que es la ineludible misión de las políticas de información.

No es que hasta antes de ese entonces no hubiera habido disposiciones del más alto nivel en los grupos sociales o las organizaciones en torno a la información, o que el surgimiento se halla dado en un rompimiento súbito e identificable entre un antes y un ahora, por el contrario, como todos los grandes momentos de la historia de la humanidad, estos se van dando de manera muy gradual, conviviendo a veces por mucho tiempo las condiciones previas y las actuales, sin embargo la presión es grande para que el acceso a la información sea universal en el menor plazo posible.

Lo que Sandra Braman plantea como un punto de quiebre es que el advenimiento de las políticas de información ha venido a ofrecer una visión integradora y una referencia obligada a todas aquellas entidades involucradas, que ella identifica en nuestra sociedad básicamente como aquellas de corte legal y económica, referencia mucho más coherente de las que se tenían previamente. Usando una frase bastante convencional pero útil, las políticas de información habrían llegado para crear una situación de ganar/ganar, en que las realidades y las ideas que anteriormente podían perderse por una falta de comunicación o de comprensión, ahora avanzarían en una misma dirección y sentido hacia su concreción.

El concepto de políticas de información es uno que ha registrado una trayectoria diferente de muchos otros de las ciencias sociales o de las ciencias de la información, en el sentido de que, al conjuntar dos términos de uso tan generalizado, cada funcionario, político, académico o teórico, tiene uno propio qué pensaría que los demás comparten, comprenden o por lo menos aceptan. Esto ha ahorrado mucho tiempo de debate estéril que deseablemente debió haberse dedicado a formular otros elementos de las políticas de la información, concretamente las relacionadas a la operativización de las mismas para el efecto lo más inmediato posible de resultados patentes a la sociedad, particularmente en la relación entre las organizaciones, los gobiernos y los ciudadanos.

Margaret Fung hace algunas claridosas consideraciones respecto a las políticas de información, entre ellas el partir de la base de que la información, siendo instrumental a la productividad, y al haberse asociado a las intenciones modernizadoras de la sociedad y al bienestar de los individuos y los grupos, tiene un carácter fundamental en el desarrollo de las naciones, la creación de políticas públicas, y los procesos de decisión y acción que se derivan de estas. Con esta premisa la progresión de pensamiento es simple y obligada, sin una cuidadosa articulación de las políticas de información con las políticas públicas, es limitado lo que podrá lograrse de ellas, ya que por sí solas y sin el refuerzo del interés de la colectividad a través de su gobierno, difícilmente podrán pasar de ser una declaración de 
buenos deseos, como ha quedado demostrado en multitud de programas sociales de incorporación a las tecnologías de la información y comunicación en todo el mundo, que quedan como oportunidades desperdiciadas.

La propia UNESCO ha declarado que la información debe ser tratada por las autoridades de cada país como uno de los recursos nacionales más preciados, la constante creación, colección, procesamiento, diseminación, transferencia, acceso, análisis y utilización de la información son indispensables para los esfuerzos del desarrollo de cualquier país, y puesto de esa manera, también de la comunidad mundial. A tal grado está llegando la compenetración de la sociedad de la información con la vida económica de los países, que en su ya clásico artículo Geza Feketekuty y Jonathan Aronson señalaban en 1984 que más del 60\% de los empleos en Estados Unidos involucraban o se relacionaban con la información, en una tendencia global que según Fung llevó a que tan solo durante la década anterior el incremento de posiciones laborales en el área hubiera pasado de diez millones a sesenta (1986), considerando niveles y posiciones que en su momento la International Labour Organization ni siquiera consideró pertinentes al formular sus expectativas de crecimiento del empleo asociado a la sociedad de la información (2004), en un ideal que en la práctica se ha proletarizado, con muy poco beneficio intelectual para quienes se desempeñan en la multitud de empleos en el amplio espectro de la provisión y manejo de información para efectos principalmente comerciales. La diferencia radica en que ahora el maquinismo ya no es en una estación en una línea de producción, sino que es frente a la pantalla de un ordenador, quizá todavía más esclavizante, y recordando una escena de 1984 de George Orwell, mucho menos liberador, pese a ser esa la supuesta pretensión.

Estas realidades demandan atención, y más que atención, la creación de políticas públicas en el ámbito de la información para que cada nación esté preparada para enfrentar una transformación de los individuos y las organizaciones que sobrepasa con mucho las previsiones de los gobiernos, sea para administrarlas, para fomentarlas, guiarlas, para democratizarlas o para contenerlas, aspecto este último que ha demostrado ser demasiado elusivo.

Lo que por mucho tiempo no representó un interés particular, ahora ha comenzado a ser estratégico para las naciones, y no solo por las preocupaciones de inteligencia y seguridad del Estado, que suelen consumir bastante esfuerzo y recursos de los gobiernos, sino de todos los sectores de la administración pública que ha adquirido consciencia, a veces demasiado súbitamente, de la importancia que tiene la información, y la ineludible exigencia que tiene de entenderse de ella mediante la herramienta básica a su alcance, las políticas públicas ya en su forma concreta de políticas de información.

Desde un punto de vista cordial, cualquier nación del mundo se podría beneficiar de la adopción del planteamiento de la UNESCO, de que la creatividad de una nación, sus visiones, sus introspecciones, son alimentadas por el análisis de la información y los datos. Con esos elementos en mano, se abre el abanico de oportunidades para formular estrategias de desarrollo con verdadero potencial de llegar a convertirse en realidad.

Tal como lo plantea Elisa Margarita Almada, en la actualidad la información es relevante para todo proceso social, así como también lo es la comprensión de los procesos de los flujos de información y su organización la mayoría de las naciones han asumido ya que las previsiones de alto nivel que hagan en torno a la información en sus sucesivas fases, son esenciales en el corto plazo para el crecimiento económico, condición no solo restringida a la economía sino también de orden político, buscada por todos los gobiernos para ofrecer a sus ciudadanos. El problema se centra ahora en el diseño de políticas de información que sean realmente efectivas para potenciar el esfuerzo individual y coordinado de los distintos actores sociales involucrados en las grandes empresas nacionales, cuando menos sobre esto, hay todas las coincidencias y acuerdos que permitirían desplantar políticas que anticiparan un cierto grado de cumplimiento.

Como lo señala Braman, coloquialmente las políticas de información proveen un paraguas para todas las acciones que tienen que ver con la información, con la comunicación y la cultura, funciones esenciales de todo gobierno nacional, de donde deriva una de las definiciones más operacionalmente útiles de lo que deberíamos entender por políticas de información para beneficiarnos de ellas: las políticas de información entendidas como el conjunto de leyes, regulaciones y posiciones doctrinales, además de la toma de decisiones y otras prácticas que tienen efectos en la constitución misma de la sociedad, involucrando la creación, proceso, flujo, acceso y uso de la información. 
Independientemente de la validez teórica y su operabilidad metodológica que le permite incorporarse con relativa facilidad a distintos ámbitos de aproximación, una definición de esta clase ofrece una oportunidad inmediata a los distintos planteamientos gubernamentales en materia de política de la información, al establecer un parámetro de comparación que deja de lado la retórica de buenas intenciones, para inquirir con fines de dar viabilidad a la acción gubernativa: ¿Hay leyes que expliciten la política de información de determinada área, hay regulación a la cual los involucrados tengan que apegarse, existen mecanismos de fomento a los que puedan acogerse, cuentan con la protección para explotar la información en sus sucesivas fases? Una política de información que contemple mínimamente estos y deseablemente muchos más, puede considerarse la guía indispensable para que una nación se adentre con ventaja en la sociedad de la información, su ausencia, implica que los esfuerzos serán por fuerza descoordinados, en una época en la que cualquier retraso, o peor cualquier desviación, tiene un fuerte impacto en la competitividad de una nación frente a otras y frente al mundo, hablando solo en términos económicos y la influencia de la economía en el potencial de bienestar del conjunto poblacional y de cada uno de los individuos.

Quizá uno de los puntos críticos en la evolución reciente de las políticas de información es la tendencia a pensar en ellas de una manera más bien aislada, sobre todo cuando las personas u organizaciones involucradas se ubican en sectores específicos de la economía y del sector productivo, como puede ser el caso de la industria informática o las empresas de telecomunicaciones. Con todo que estas importantes ramas de la economía merecen el adecuado tratamiento por parte de la administración pública de cada país, las políticas de información no se restringen a una cuestión estrictamente productiva, la visión debe ampliarse para abarcar la totalidad de la población y los sectores que la afectan, pues es el conjunto de los habitantes de una nación, y para el caso del mundo entero, los que pueden y deben beneficiarse de la información.

Desde este punto de vista, las políticas de información se inscriben como una parte trascendental de las políticas públicas, y dado el carácter que han ido adquiriendo y casi acaparando como motor del progreso económico en la sociedad de la información, necesariamente deben pensarse como un elemento articulado a los grandes proyectos y programas nacionales de desarrollo, particularmente de lo que en algunos países latinoamericanos se denomina desarrollo social, y que abarca entre otros muchos y muy vastos ámbitos, los del mejoramiento de la calidad de vida y la educación.

En palabras de Guerra y Jordán, las tecnologías de información, para las que se desarrollan las políticas de información, y no como quizá debería ser al revés, son recursos de orden general que al ofrecer la versatilidad de poder incorporarse a cualquier sector productivo y social, optimizando las actividades cotidianas, se hace urgente la masificación del acceso a ellas, propugnando por su adopción en todos los ámbitos económicos para que de esa manera se pueda aprovechar el potencial de una sociedad basada en la información y el conocimiento, proceso en el cual el gobierno tiene una responsabilidad innegable de conducción (2010).

\section{Políticas de información y bibliotecas}

Puede hablarse ya de una tendencia a nivel global, de las políticas de información como parte de las políticas públicas, así lo pone de manifiesto la Declaración de la IFLA sobre bibliotecas y desarrollo, signada en agosto del 2013 en Singapur. El organismo internacional recomienda a los generadores de políticas públicas y a las personas que ocupan posiciones que orientan las prácticas gubernamentales estratégicas, a reconocer el papel del acceso a la información como un elemento fundamental para apoyar el desarrollo; a reconocer el rol de las bibliotecas y de los bibliotecarios como auténticos agentes del desarrollo; y a animar a los estados miembros de la Organización de las Naciones Unidas para brindar su respaldo al marco que considera la información como sustento del desarrollo, a través de la provisión de redes, información y recursos humanos, principalmente las bibliotecas y otras instituciones de interés público (IFLA, 2013).

Para los gobiernos de muchas, si no es que de la totalidad de las naciones representadas en el la UNESCO, en la IFLA y por supuesto en la Organización de las Naciones Unidas, el acceso a la información es un derecho humano fundamental, la cual puede y debe ser vista como un instrumento para romper el ciclo de pobreza en el nivel individual y familiar, al ser potencialmente el principal sustento del desarrollo. La IFLA sostiene que la biblioteca es el único espacio en el cual la gente que habita en las comunidades puede tener acceso a la información que le permita 
mejorar su educación, adquirir y mejorar sus habilidades, encontrar empleo, tomar decisiones informadas y ganar consciencia sobre temas ambientales, entre un ilimitado número de otras posibilidades (IFLA, 2013).

Para la organización, como también es de suponer para los representantes de los países que la integran, la biblioteca tiene o podría llegar a tener un papel único, el de socio para el desarrollo, al proveer acceso a información en todos los formatos, y al ofrecer servicios y programas que satisfacen las necesidades concretas de información a la población, en un ambiente muy complejo y de rápido cambio. La IFLA considera el momento presente como una oportunidad única para asignar a las bibliotecas funciones específicas dentro del marco de las políticas de información de cada nación, pues al estar cerca el vencimiento de los objetivos que la comunidad de naciones se planteó para el horizonte 2015, los gobiernos nacionales pueden transformar a esta institución en un mecanismo confiable como pocos, para garantizar el éxito de sus programas de desarrollo.

Contrario a las tecnologías de información y comunicación, y el papel que han tenido estas como detonador del nacimiento de las políticas de información, las bibliotecas por sí mismas y como parte de los sistemas educativos de las distintas sociedades, son entidades que no solo se están sumamente extendidas en términos de cobertura geográfica, sino que son también de las más estables y permanentes. Por esta razón, y por qué es el medio natural para el acceso a la sociedad de la información para amplios sectores de población que por su grado de marginación, o por la falta de infraestructura de conectividad, no pueden acceder a Internet por una vía diferente, la biblioteca resurge como una opción de primera magnitud para la implementación, tanto de las políticas públicas del gobierno nacional, como de las políticas de información.

En la Declaración de Singapur la IFLA lo pone en términos bastante claros, las bibliotecas son entidades sociales de corte eminentemente humanista que tienen la misión y están en capacidad de proveer oportunidades para todos. Sin importar distinciones que ocurren cotidianamente en otros espacios, la biblioteca ha procurado pasar por alto diferencias de raza, nacionalidad, origen étnico, género, preferencia sexual, edad, religión, situación económica, e incluso hace esfuerzo sustancial para satisfacer a grupos de personas con discapacidades. Difícilmente los gobiernos nacionales, hablando de su responsabilidad para la formulación y cumplimiento de las políticas de información, estarán en posibilidad de encontrar otra institución con mayor capacidad para bajar aquellas del elevado nivel del discurso, al de la práctica diaria en todos los rincones de la geografía y para toda la población (IFLA, 2013).

Continuando con la idea, si la sociedad de la información tiene como una de sus premisas primordiales el del valor económico que pueda desprenderse del acceso, dominio y utilización de la información, la biblioteca ha funcionado durante décadas y hasta siglos como un factor de igualación social, al ofrecer a los segmentos más vulnerables y desprotegidos acceso a materiales que no podrían encontrar en ningún otro sitio, si las políticas públicas, las de economía y las de desarrollo social contemplan la inclusión de todos, también deberán hacerlo las políticas de información, preferentemente a través de la educación y la biblioteca, dos sistemas que además de su amplia extensión, son algunos sobre los que puede ejercer la mayor supervisión y control.

Otro elemento que consideramos relevante es el de que aunque la tendencia del mundo actual es a exaltar las bondades de los gobiernos democráticos, las políticas públicas, y particularmente las políticas de la información no suelen verse desde una perspectiva participativa, literalmente, democrática. En efecto, los gobiernos actuales siguen apegándose al paradigma de que la única función del ciudadano para con la democracia es el sufragar en las urnas, y poco más; este modelo de interacción no reza con la sociedad de la información, la cual a como se ha venido dando, más que porque así haya sido concebida, todos los actores tienen igual voz y voto. Cabe señalar que para la formulación de las políticas de información, se suele tener muy poco en cuenta las opiniones de la gran masa de población, construyéndose por lo general con las opiniones expertas de los funcionarios públicos y las de grupos de interés, lo que no es extraño que derive en una falta de atención a amplios sectores.

Las bibliotecas, salvo muy escasas excepciones, no han sido tenidas como interlocutores válidos para la redacción de políticas nacionales de información. En parte esto se debe al propio aislamiento que tienen respecto de los sistemas educativos y de otras funciones de gobierno, y en parte porque desde el poder público no han sido calificadas cono "stakeholders" con peso, esto a pesar de que por el número de personas a las que suelen atender para ofrecerles precisamente la información que demandan, las bibliotecas representan a mucha gente, pero no solo eso, estas entidades son las que mejor conocimiento tienen de la clase de materiales que se necesitan, y en qué 
condiciones deben ser preferentemente entregados. Cegarse a esta clase de interlocución es cerrar la puerta a políticas de información funcionales y efectivas, y que realmente sirvan como factor de desarrollo.

\section{Conclusiones}

Poca duda cabe de que las políticas de información constituyen una tendencia orientadora para el desarrollo de las tecnologías de información y comunicación en el mundo, pero esto no quiere decir que ellas mismas cuenten con una orientación satisfactoria desde la perspectiva de las grandes masas de población.

Como tales, las políticas de información han sido impulsadas mayormente por parte de los grupos de interés económico, que han presionado a los gobiernos nacionales a plantear o en el peor de los casos, a adoptar algunas que favorecen a los citados grupos.

Si bien la retórica de muchos gobiernos cita las ideas correctas: educación, desarrollo humano, equidad, crecimiento económico, y bienestar social, entre otros, ligándolos a las políticas de información emprendidas o por emprender, estas con escasas excepciones fallan en bajar al nivel de la puesta en práctica de la política de Estado, esencial para garantizar el cumplimiento y el éxito cuantificable de su aplicación.

En especial la relación entre política de información y la institución bibliotecaria, que podría considerarse obvia y obligada para quienes plantean metas y disponen de los recursos para ella, rara vez aparecen más allá del discurso, el resultado es que la infraestructura permanece sin usar, y sus usuarios al margen de la tendencia de la sociedad de la información.

La recomendación es simple, incluir a la biblioteca como concepto y en sus diversos tipos y acepciones, como el recurso de contacto entre las políticas de información y la población que deberá beneficiarse de ellas.

\section{Bibliografía}

Almada Navarro, E. (2012) Bases teóricas para comprender las Políticas de Información, en La naturaleza objetiva y subjetiva de las Políticas de Información http://132.248.242.6/ publica/conmutarl.php?arch=3\&idx=147

Braman, S. (2011). Defining Information Policy, Journal of Information Policy, 1. http://iip.vmhost.psu.edu/ojs/index.php/iip/article/viewFile/19/14

Fung, M. (1986) National Information Policy, some basic considerations http://jlis.glis.ntnu.edu.tw/ojs/index.php//lis/article/download/194/194

Feketekuty, G. and Aronson, J. D. (1984), Meeting the Challenges of the World Information Economy. World Economy, 7: 63-86. http://onlinelibrary.wiley.com/doi/10.1111/i.1467-9701.1984.tb00258.x/abstract

Guerra, M, Jordán, V. (2010) Políticas públicas de sociedad de la Información en América Latina: ¿una misma visión?, ONU, Comisión Económica para América Latina y el Caribe.

IFLA Statement on Libraries and Development (2013)

http://www.ifla.org/files/assets/alp/statement on libraries and development.pdf

International Labour Organization (2004). Conclusions on the future of work and quality in the information society, http://www.ilo.org/wcmsp5/groups/public/@dgreports/@dcomm/documents/meetingdocument/kd00065.pdf 


\section{Datos del autor}

Horacio Cardenas Zardoni

Magister y candidato a Doctor en Bibliotecología y Estudios de la Información por la Universidad Nacional Autónoma de México, responsable de Fomento y Difusión y Gestión de Calidad del Sistema de Infotecas Centrales de la Universidad Autónoma de Coahuila. Cultiva las líneas de investigación: sociedad de la información, políticas de información, financiamiento a bibliotecas, temas en los que ha publicado variedad de artículos en revistas en América Latina y España, es autor de los libros: "El humanismo en las ciencias desde la perspectiva del académico universitario", "Entorno Digital y futuro de la cultura", y "Cultura del Carbón".

zardoni@uadec.edu.mx

$\begin{array}{ll}\text { Recibido-Received } & : 2013-11-08 \\ \text { Aceptado-Accepted } & : 2013-12-30\end{array}$

\section{(oc) EY}

This work is licensed under a Creative Commons Attribution 4.0 United States License.

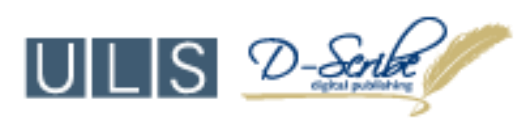

This journal is published by the University Library System of the University of Pittsburgh as part of its $\underline{D}$-Scribe Digital Publishing Program and is cosponsored by the University of Pittsburgh Press. 